\title{
Zero-forcing Electrical Filters for Direct Detection Optical Systems
}

\author{
Peter C. Li and Pierre A. Humblet \\ MIT, 35-425, Cambridge, MA 02139, USA, and Eurecom, B.P. 193, 06904 Sophia Antipolis, France
}

\begin{abstract}
Intersymbol interference in direct detection optical systems can limit the channel spacing in frequency division multiplexing and prevent multilevel signaling. We investigate a zero-forcing electrical filter to cancel intersymbol interference and compare its performance with a matched filter and a rectangular response flter, for $M$-ary amplitude modulation.
\end{abstract}

\section{MODEL}

In our model, the receiver front-end is composed of an optical filter, a photodetector and a low-pass electrical filter as shown in Figure 1. The photodiode is modelled as a square-law de-

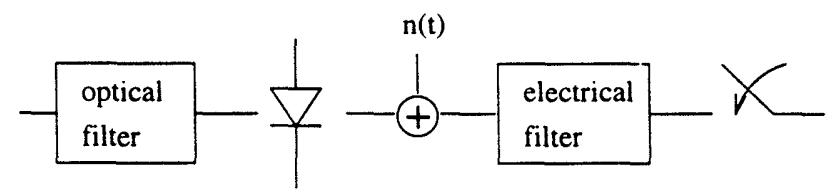

Figure 1: The receiver front-end includes an optical filter, a photodiode and a low-pass electrical filter followed by a baud rate sampler.

vice whose output is proportional to the magnitude square of the received signal envelope. The thermal noise $n(t)$ from the electronics is assumed to be the dominant noise and is modelled as additive white Gaussian noise.

\section{Zero-ForCING FILTERS}

Without loss in generality, we design the filter for sampling time $t=0$. The output of the electrical filter at this time is

$$
\sum_{i} \sum_{j} a_{i} a_{j} \int p_{i, j}(\sigma) h(\sigma) d \sigma+N,
$$

where $h(t)$ is the time-reversed impulse response of the electrical filter, $p_{i, j}(t)=\operatorname{Re}\left\{p(t-i T) p^{*}(t-j T)\right\}, p(t)$ is the complex envelope of the received optical pulse taking into account the transmit pulse and the channel response, $p^{*}(t)$ is the complex conjugate of $p(t), a_{k}$ (taken as real here) is the $k$ th transmit amplitude, and $N$ is the noise at the sampled output. We write the integral $\int p_{i, j}(t) h(t) d t$ as an inner product $\left\langle p_{i, j}, h\right\rangle$ where $\langle x, y\rangle \equiv \int x(t) y(t) d t$.

We define the ISI space, $I$, as the space spanned by $p_{i, j}(t)$ 's without $p_{0,0}(t)$, the desired signal. The signal space is defined as the space spanned by $I$ and $p_{0,0}(t)$. A filter $h(t)$ is a zeroforcing filter if the sampled output has no ISI, i.e. we want the output of $h(t)$ to depend only on $a_{0} a_{0}$ at sampling time $t=0$. A necessary and sufficient condition for $h(t)$ to be a zero-forcing filter is

$$
\begin{aligned}
& \left\langle p_{i, j}, h\right\rangle \neq 0, \text { if } i=j=0, \text { and } \\
& \left\langle p_{i, j}, h\right\rangle=0, \text { otherwise. }
\end{aligned}
$$

For filters that satisfy (2), we are interested in the one that minimizes the noise variance when $\left\langle p_{0,0}, h\right\rangle$ is set equal to a constant. The time-reversed impulse response of this filter, if it exists, is proportional to the component of $p_{0,0}(t)$ orthogonal to $I$.

\section{FABRY-PEROT INTERFEROMETER}

We consider a Fabry-Perot filter as the optical demultiplexing filter [1]. The envelope of the impulse response is well approximated by $\frac{1}{\tau} e^{-t / \tau}$ for $t \geq 0$. The envelope of the transmit pulse is equal to 1 in $\left[0, \frac{\log _{2} M}{R}\right]$ and 0 otherwise, where $M$ is the number of signaling levels and $R$ is the bit rate. The symbol set is equal to $\left\{\sqrt{\frac{i}{M-1}} \mid 0 \leq i \leq M-1\right\}$. We keep the bit rate fixed and plot the normalized eye opening for different $\tau^{\prime}$, where $\tau^{\prime}=\tau R$ is a parameter proportional to bandwidth efficiency in units of $(b / s / H z)$. The normalized eye opening is the vertical opening of an eye-diagram when the electrical filter has unit energy. We consider a filter matched to the postdetection electrical pulse $p_{0,0}(t)$ (single pulse transmitted), the minimum noise variance zero-forcing filter, and a filter with rectangular impulse response on $\left[0, \frac{\log _{2} M}{R}\right]$. For the rectangu-

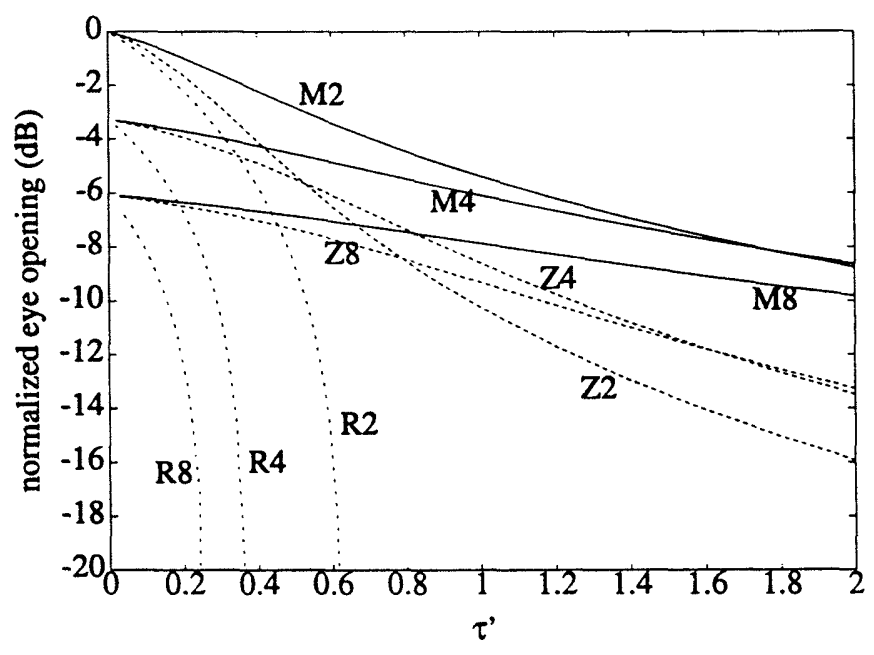

Figure 2: The optical filter is a Fabry-Perot interferometer. The curves are labelled as follows: (M) matched filter curve, $(Z)$ zero-forcing filter, $(R)$ rectangular filter, and $(2,4,8)$ level signaling.

lar filter, there is no advantage to multilevel signaling, same as in [1]. On the other hand the zero-forcing filter performs much better and there is advantage to four level signaling for large values of $\tau^{\prime}$.

\section{ACKNOWLEDGEMENTS}

This work was supported by NSF grant $9206379-$ NCR.

\section{REFERENCES}

[1] L. Cimini and G. Foschini. Can multilevel signaling improve the spectral efficiency of ask optical fdm systems? IEEE Transactions on Communications, 41(7):1084-1090, July 1993. 